\title{
Two-Member Markov Processes toward an Equilibrium from a Continuum of Initial States
}

\author{
Jinsik $\mathrm{MOK}^{1, *}$ and Hyoung-In $\mathrm{LEE}^{2, *}$ \\ ${ }^{1}$ Department of Industrial and Management Engineering, Sunmoon University, \\ Tangjeong-myeon, Asan-si, Chungnam 31460, Korea \\ ${ }^{2}$ Research Institute of Mathematics, Seoul National University, 599 Gwanak-ro, Gwanak-gu, Seoul 08826, Korea
}

\begin{abstract}
Dynamics of two-member Markov processes is formulated based on the binomial probability. Sets of initial states are then sought such that the final state reaches an equilibrium. On the two-parameter phase plane, such initial states are found to exhibit diverse geometric configurations depending on the source probability. Those initial-state boundaries undergo phase transitions ranging over pills, stripes, circles, ellipses, lemons, and even fuzzy shapes. These results are quite helpful in understanding several physical phenomena involving photons, electrons, and atoms. For convenience of discussion, deformations of vortices are taken as an example.
\end{abstract}

KEYWORDS: Markov process, binomial probability, geometric configuration, initial state, phase transition, vortex

\section{Introduction}

Based on the binomial probability [1], we are here to examine certain Markov processes from two perspectives: geometric deformations [2], and two-member interactions [3].

Firstly, consider geometric deformations of two-dimensional vortices as an example [2]. There is a vast literature on vortices as a physical phenomenon of either electronic or photonic nature [3]. The circular shape represented by typical vortices is just one of many possible geometric configurations. For instance, a slight material anisotropy alters the circular shape of a vortex into varying degrees of elliptic shapes [4]. We could even suppose that a linear stripe on a plane is deformed eventually into a circular vortex after undergoing various dynamical evolutions. One of the key characters of vortices is the direction of circulation. For instance, vortices of opposite senses are more likely to cancel among them, thus possibly contributing to a net zero circulation [3]. In terms of the aforementioned stripe, the rotational direction of a vortex corresponds to which way information is propagated along the stripe's direction. This discussion on vortices has been made in both physical real space and parameter space.

Secondly, consider a two-member interaction which is the simplest one among multiple members. As an example, many issues in quantum optics are discussed in terms of two-member systems [5,6]. As another example, two-mode radiation field treats a quantum mechanical vortex state when generated by subtracting a photon [7]. Notice that the vortex states in [7] are represented by two-mode wave functions on the parameter plane formed by the two quadrature coordinates. The complex parameter representing the inter-mode interaction of a squeezed state plays a key role in determining how elliptic vortex states occur [7]. Besides, the phase plane can be what is employed for the Wigner function $[5,8]$.

As yet another two-member system, consider interactions between electromagnetic field and a two-level atom $[5,6,8]$. Such two-level systems form the basis for the interferometers [9-13]. In addition, the Schwinger boson representation for even-numbered spin systems is admissible, thus revealing a bosonic feature of the collective ensemble of fermionic atoms [11]. A similar pseudospin picture is employed for the two-dimensional electrons of a bilayer graphene or a double quantum well under the action of perpendicular magnetic fields [3]. Additionally, two spin-rotation angles serve to form a phase plane in case with quantum walks [2].

Let us combine the aforementioned two aspects of geometric deformations and two-member interactions. In our study, two parameters specify an initial state of a certain dynamics. The dynamics itself is constructed by two-member binomial probability with the source parameter acting as a time-like variable $[1,8]$. Our Markov process is hence established by employing various combinations of such binomial probabilities in forming pertinent transition probability matrices $[5,6,13,14]$. The end state of this Markov process is assumed to be in equilibrium where its constituent substates are of equal probabilities. By this way, we can impose a certain constraint on the initial state. Our discussion will center on the various phase-plane shapes for such an initial state. 
As a result, such initial-state boundaries on the initial-state phase plane are found to range over medicine pills, linear stripes, circles, ellipses, lemons, and fuzzy shapes as the source probability is altered. In particular, phase transitions are found to take place between different geometric shapes with the source probability playing the role of a critical parameter [2]. It turns out that the source probabilities of one third and two thirds delineate drastic changes in the shapes of those initial-state boundaries. Elongated ellipses on the frequency space are also seen for interference patterns of light polarization $[12,13]$. In a few physical examples observed in real space, we find a similar array of shape changes with varying parameters $[5,10]$. These phenomena on two-dimensional real space are of particular importance from the viewpoint of phase transitions and topological physics [2, 3, 13].

Consider a few more characteristics of Markov processes. The approximation of adiabatic slow processes underlies Markov processes $[3,14,15]$. Therefore, non-Markovian processes involving fast optical pulses or switching are not under our investigation [6,8]. In addition, our Markov process corresponds to the steady increase in indistinguishability among the member states $[9,10,12,13]$. Along the same line of reasoning, Markov processes governed by random transition matrices normally erase the initial-state dependence as events progress [5]. In stark contrast, the initial states are usually remembered whenever collapses and revivals are prevalent.

This paper is organized as follows. In Sect. 2, fundamentals of Markov processes are presented for a single member. In Sect. 3, Markov processes are worked out for two members. In Sect. 4, the initial-state boundaries are investigated for a simpler two-member system. In Sect. 5, phase changes are discussed for a more complicated system as the source probability is varied. Section 6 presents discussions followed by conclusion in Section 7.

\section{Fundamentals of Markov Processes}

Consider the binomial probability defined below [1].

$$
C_{n}^{g}=\frac{g !}{n !(g-n) !}, \quad P_{n}^{g}(q)=C_{n}^{g}(1-q)^{g-n} q^{n} .
$$

In terms of the balls-in-boxes argument, the integers $n$ and $g$ refer to the numbers of indistinguishable balls and boxes, respectively. Alternatively, $n$ is called a generation number, whereas $g$ is called the member or particle number. Therefore, the total of admissible substates is $g+1$. The source probability $q$ is continuous over the interval $0 \leq q \leq 1$. Furthermore, $q$ can be interpreted from several perspectives [3,7,8,11-14]: (i) the probability of rightward or leftward one-dimensional random walks, (ii) the doping level or degree of defects in compound materials, (iii) a dynamical evolution variable, or (iv) squeezing parameter. By incorporating an additional time variable into $P_{n}^{g}(q)$, evolutionary dynamics can be readily formulated $[1,5,8]$.

Figure 1 displays both $P_{n}^{1}(q)$ in (a) and $P_{n}^{2}(q)$ in (b), both plotted against $q$. Here, $P_{0}^{1}(q)=1-q$ and $P_{1}^{1}(q)=q$, whereas $P_{0}^{2}(q)=(1-q)^{2}, P_{1}^{2}(q)=2(1-q) q$, and $P_{1}^{1}(q)=q^{2}$. At $q=1 / 2$, the symmetry of $P_{1}^{2}(q)$ with respect to $q$ is clearly visible from Fig. 1(b) $[1,11]$.

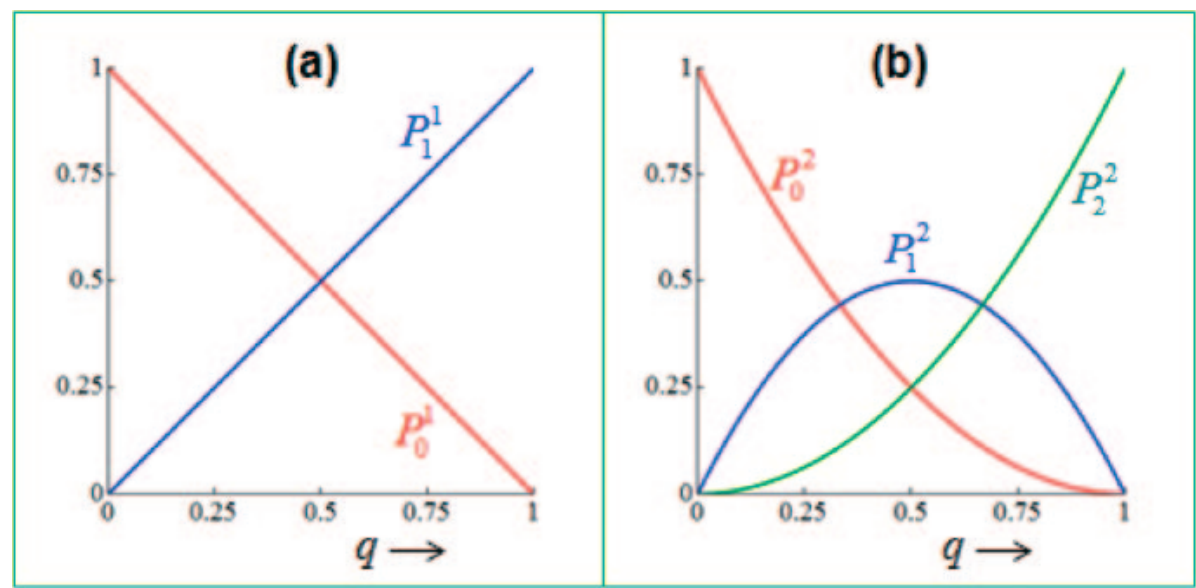

Fig. 1. The binomial probability for a single particle with $g=1$ in (a), and two particles with $g=2$ in (b).

Let us consider the simplest case with $g=1$. The transition probability matrices (TPMs) based on $P_{n}^{1}(q)$ have two possibilities as follows.

$$
T_{0}^{1} \equiv\left(\begin{array}{cc}
P_{0}^{1} & P_{1}^{1} \\
P_{0}^{1} & P_{1}^{1}
\end{array}\right), \quad T_{1}^{1} \equiv\left(\begin{array}{cc}
P_{0}^{1} & P_{1}^{1} \\
P_{1}^{1} & P_{0}^{1}
\end{array}\right)
$$


Here, the subscript $m$ in $T_{m}^{1}$ indicates different ways of permutation. Suppose that $T_{m}^{1}$ gives rise to a pair of eigenvalues $\left(\lambda_{m}^{1}\right)_{\sigma}$ with $\sigma=0,1$. As one limitation on Eq. (2), only the amplitude aspect of dynamics is taken into account without its phase information.

Let the $k$-th Markov step be denoted by the column vector $\vec{V}(k) \equiv\{x(k), y(k)\}^{T}$. Therefore, an initial state is given by $\vec{V}(0) \equiv\{x(0), y(0)\}^{T}$. For the first case with TPM $T_{0}^{1}$, we find the two eigenvalues $\left(\lambda_{0}^{1}\right)_{0}=1$ and $\left(\lambda_{0}^{1}\right)_{1}=0$. Hence, $x(k)=y(k)=(1-q) x(0)+q y(0)$. As a result, $\vec{V}(k)$ remains the same with varying $k$ throughout a given Markov process for any combinations of $x(0)$ and $y(0)$. In addition, a fixed-point state $x(k)=y(k)=1 / 2$ is established for the particular initial state $x(0)=y(0)=1 / 2$.

In the second case with $T_{1}^{1}$, we have $\left(\lambda_{1}^{1}\right)_{0}=1$ and $\left(\lambda_{1}^{1}\right)_{1}=1-2 q$. From both left and right eigenvectors [1], its Markov process is described by the following transient dynamics [10].

$$
\vec{V}(k) \equiv\left\{\begin{array}{l}
x(k) \\
y(k)
\end{array}\right\}=\frac{1}{2}\left\{\begin{array}{l}
1 \\
1
\end{array}\right\}+\frac{(2 q-1)^{k}}{2}\left(\begin{array}{cc}
1 & -1 \\
-1 & 1
\end{array}\right)\left\{\begin{array}{l}
x(0) \\
y(0)
\end{array}\right\} .
$$

Here on the right-hand side, the first term refers to the stationary state, being constant in this particular case. In the meantime, the second term means transient or decaying dynamics since $|2 q-1|<1$ for all $q$ over $0<q<1$. A special is obtained exactly for $q=1$ such that $x(k)=\frac{1}{2}+\frac{1}{2} x(0)-\frac{1}{2} y(0)$ and $y(k)=\frac{1}{2}-\frac{1}{2} x(0)+\frac{1}{2} y(0)$ for any values of $k$. As a check, we obtain $x(k)+y(k)=1$ for all $k$, of course. There is no oscillatory feature with varying $k$ in this one-member case, due to lack of interactions.

\section{Two-Member Markov Processes}

As the next simple case, consider the case with $g=2$. The corresponding six TPMs $T_{m}^{2}$ are defined as follows.

$$
T_{0}^{2} \equiv\left(\begin{array}{ccc}
P_{0}^{2} & P_{1}^{2} & P_{2}^{2} \\
P_{0}^{2} & P_{1}^{2} & P_{2}^{2} \\
P_{0}^{2} & P_{1}^{2} & P_{2}^{2}
\end{array}\right), \quad T_{1}^{2} \equiv\left(\begin{array}{ccc}
P_{0}^{2} & P_{1}^{2} & P_{2}^{2} \\
P_{1}^{2} & P_{0}^{2} & P_{2}^{2} \\
P_{2}^{2} & P_{1}^{2} & P_{0}^{2}
\end{array}\right), \quad T_{2}^{2} \equiv\left(\begin{array}{ccc}
P_{0}^{2} & P_{1}^{2} & P_{2}^{2} \\
P_{2}^{2} & P_{0}^{2} & P_{1}^{2} \\
P_{0}^{2} & P_{2}^{2} & P_{1}^{2}
\end{array}\right) .
$$

Likewise, the remaining TPMs $T_{3}^{2}, T_{4}^{2}$, and $T_{5}^{2}$ can be easily constructed. Let us now denote the triplet of eigenvalues by $\left(\lambda_{m}^{2}\right)_{\sigma}$ with $\sigma=0,1,2$ for each of $T_{m}^{2}$. Furthermore the $k$-th Markov step is denoted by the column vector $\vec{V}(k) \equiv$ $\{x(k), y(k), z(k)\}^{T}$ with the corresponding initial state $\vec{V}(0) \equiv\{x(0), y(0), z(0)\}^{T}$.

First, consider the simplest TPM $T_{0}^{2}$, for which three eigenvalues are $\left(\lambda_{0}^{2}\right)_{0}=1,\left(\lambda_{0}^{2}\right)_{1}=0$, and $\left(\lambda_{0}^{2}\right)_{2}=0$. Correspondingly, the stationary Markov process is obtained as follows.

$$
\vec{V}(k) \equiv\left\{\begin{array}{c}
x(k) \\
y(k) \\
z(k)
\end{array}\right\}=\left(\begin{array}{ccc}
(1-q)^{2} & 2(1-q) q & q^{2} \\
(1-q)^{2} & 2(1-q) q & q^{2} \\
(1-q)^{2} & 2(1-q) q & q^{2}
\end{array}\right)\left\{\begin{array}{c}
x(0) \\
y(0) \\
z(0)
\end{array}\right\} .
$$

Hence, we have $x(k)=y(k)=z(k)$ for any $k$ with $x(k)=(1-q)^{2} x(0)+2(1-q) q y(0)+q^{2} z(0)$. Besides, we find the fixed-point state $x(k)=y(k)=z(k)=1 / 3$ for the particular initial state $x(0)=y(0)=z(0)=1 / 3$.

Next, consider the non-trivial TPM $T_{1}^{2}$, for which three eigenvalues are $\left(\lambda_{1}^{2}\right)_{0}=1$, $\left(\lambda_{1}^{2}\right)_{1}=1-2 q$, and $\left(\lambda_{1}^{2}\right)_{2}=1-4 q+3 q^{2}$. Firstly, $\left(\lambda_{1}^{2}\right)_{1}$ vanishes at $q=1 / 2$. Secondly, we rewrite the third eigenvalue such that $\left(\lambda_{1}^{2}\right)_{2}=3(q-2 / 3)^{2}-1 / 3$. Summarizing the respective ranges of the eigenvalues,

$$
\left\{\begin{array} { l } 
{ ( \lambda _ { 1 } ^ { 2 } ) _ { 1 } \equiv 1 - 2 q } \\
{ ( \lambda _ { 1 } ^ { 2 } ) _ { 2 } \equiv 1 - 4 q + 3 q ^ { 2 } }
\end{array} \Rightarrow \left\{\begin{array}{c}
0<\left(\lambda_{1}^{2}\right)_{1}<1 \\
-1 / 3<\left(\lambda_{1}^{2}\right)_{2}<1
\end{array} .\right.\right.
$$

The resulting Markov process is found as follows.

$$
\vec{V}(k) \equiv\left\{\begin{array}{c}
x(k) \\
y(k) \\
z(k)
\end{array}\right\}=A_{1}^{2}(q)\left\{\begin{array}{c}
x(0) \\
y(0) \\
z(0)
\end{array}\right\} .
$$

Here, the Markov dynamics $A_{1}^{2}(q)$ for the TPM $T_{1}^{2}$ is broken first into three parts $A_{1}^{2}(q) \equiv\left[A_{1}^{2}(q)\right]_{0}+\left[A_{1}^{2}(q)\right]_{1}+\left[A_{1}^{2}(q)\right]_{2}$ as follows.

$$
\begin{aligned}
A_{1}^{2}(q) \equiv & {\left[A_{1}^{2}(q)\right]_{0}+\left[A_{1}^{2}(q)\right]_{1}+\left[A_{1}^{2}(q)\right]_{2} } \\
& +\frac{1}{8-6 q}\left(\begin{array}{lll}
4-6 q+3 q^{2} & 4-4 q & 4 q-3 q^{2} \\
4-6 q+3 q^{2} & 4-4 q & 4 q-3 q^{2} \\
4-6 q+3 q^{2} & 4-4 q & 4 q-3 q^{2}
\end{array}\right)
\end{aligned}
$$




$$
+\frac{(1-2 q)^{k}}{q+2}\left(\begin{array}{ccc}
q & 0 & -q \\
q & 0 & -q \\
q-2 & 0 & -q
\end{array}\right)+\frac{\left(1-4 q+3 q^{2}\right)^{k}}{4-3 q}\left(\begin{array}{ccc}
2-2 q & 2 q-2 & 0 \\
q-2 & 2-q & 0 \\
2-2 q & 2 q-2 & 0
\end{array}\right) .
$$

Let us examine the right-hand side of Eq. (8). The first term $\left[A_{1}^{2}(q)\right]_{0}$ refers to the stationary state [5]. Both second term $\left[A_{1}^{2}(q)\right]_{1}$ and third term $\left[A_{1}^{2}(q)\right]_{2}$ indicate transient dynamics because of Eq. (6). It is worth noting that the end value of $q=1$ leads to infinitely oscillatory dynamics, because of $(1-2 q)^{k} \rightarrow(-1)^{k}$ in $\left[A_{1}^{2}(q)\right]_{1}$. This oscillation is in stark contrast to the previous one-member case discussed by Eq. (3), where $(2 q-1)^{k} \rightarrow(+1)^{k}=1$ for $q=1$ even with varying $k$.

\section{Initial-State Boundaries for an Equilibrium Stationary State}

Let us focus on the following stationary term on the right-hand side of Eq. (8) obtained as $k \rightarrow \infty$.

$$
\vec{V}(\infty) \equiv\left\{\begin{array}{c}
x(\infty) \\
y(\infty) \\
z(\infty)
\end{array}\right\}=\frac{1}{8-6 q}\left(\begin{array}{ccc}
4-6 q+3 q^{2} & 4-4 q & 4 q-3 q^{2} \\
4-6 q+3 q^{2} & 4-4 q & 4 q-3 q^{2} \\
4-6 q+3 q^{2} & 4-4 q & 4 q-3 q^{2}
\end{array}\right)\left\{\begin{array}{l}
x(0) \\
y(0) \\
z(0)
\end{array}\right\} .
$$

The central question now reads: "When is the set of initial states leading to the equilibrium stationary state?". This happens if the equilibrium condition $x(\infty)=y(\infty)=z(\infty)=1 / 3$ of equal probabilities is satisfied, whereby Eq. (9) leads to the following constraint.

$$
\frac{\left(4-6 q+3 q^{2}\right) x(0)+(4-4 q) y(0)+\left(4 q-3 q^{2}\right) z(0)}{8-6 q}=\frac{1}{3} .
$$

Since $x(0)+y(0)+z(0)=1$ according to the probability constraint, the initial-state vector $\vec{V}(0)$ can be parameterized as follows by two spherical angles $(\theta, \phi)$ over a spherical surface of unit radius $[2,3,10,11,15,16]$.

$$
\vec{V}(0) \equiv\left\{\begin{array}{c}
x(0) \\
y(0) \\
z(0)
\end{array}\right\}=\left\{\begin{array}{c}
\cos ^{2} \theta \\
\sin ^{2} \theta \cos ^{2} \phi \\
\sin ^{2} \theta \sin ^{2} \phi
\end{array}\right\} .
$$

In terms of the two angles $(\theta, \phi)$ in Eq. (11), the requirement in Eq. (10) is cast into the following.

$$
\begin{aligned}
0=f_{1}^{2}(\theta, \phi ; q) \equiv & \left(4-6 q+3 q^{2}\right) \cos ^{2} \theta+(4-4 q) \sin ^{2} \theta \cos ^{2} \phi \\
& +\left(4 q-3 q^{2}\right) \sin ^{2} \theta \sin ^{2} \phi-(8-6 q) / 3 .
\end{aligned}
$$

Let us call $f_{1}^{2}$ the "residual function", which is desired to vanish. Figure 2 shows $f_{1}^{2}$ on two kinds of parameter planes. Firstly, the three panels (a3)-(a5) enclosed by the horizontal green box display $f_{1}^{2}(x(0), y(0) ; q)$ on the $x(0)-y(0)$-plane. Secondly, the five panels (b1)-(b5) enclosed by the two-row red box plot $f_{1}^{2}(\theta, \phi ; q)$ on the $(\theta, \phi)$-plane. Here, both $\theta, \phi$ coordinates are normalized by $\pi$ so that the $(\theta, \phi)$-plane is searched over the rectangular domain $\{(\theta, \phi): 0 \leq \theta \leq \pi, 0 \leq \phi \leq \pi\}$.

The source probability $q$ is specified on the upper portion of each panel except for panel (b0). We prepared both panels (a3) and (b3) with the equal $q=0.25$, Likewise, both panels (a4) and (b4) are drawn with the equal $q=0.6665$. In addition, both panels (a5) and (b5) are plotted with the equal $q=0.999$. Panels (b1) and (b2) are made with $q=0$ and 0.1 in order to provide a more detailed picture on the $(\theta, \phi)$-plane. For panels (b1)-(b5), an arrow is inserted between two neighboring panels to show the direction of increasing $q$. The panel (b0) will be explained shortly.

On each panel, the brighter and darker colors refer to $f_{1}^{2}>0$ and $f_{1}^{2}<0$, respectively. In particular, the strength of the bluish color corresponds to the magnitude $\left|f_{1}^{2}\right|$ for the case $f_{1}^{2}<0$. As a visual guide to readers, the positive and negative values on each panel are indicated by the signs "+" and "-", respectively. Therefore, the boundary(ies) between the two zones of different colors indicate(s) the set of initial states leading to the same equilibrium stationary state.

Consider panels (a3)-(a5) of Fig. 2 plotted for $q=0.25,0.6665,0.999$, respectively. Recall however that the state at $q=1$ is not reachable in a finite value of the time-like parameter $q$ in reference to Eq. (6). It is why we prepared panel (a5) for $q=0.999$ instead of $q=1$. The boundary on this $x(0)-y(0)$-plane is found to be a single straight line on all the three panels. Consider the equilibrium initial state [11]: $x(0)=y(0)=z(0)=1 / 3$. In this case, Eq. (9) gives rise to $x(\infty)=y(\infty)=z(\infty)=1 / 3$, a trivial result, but confirming again that the equilibrium initial state is maintained. We have marked this special state $x(\infty)=y(\infty)=z(\infty)=1 / 3$ by the single small disk in red circular boundary on panels (a3)-(a5). These small disks are a little bit off the linear boundary because of the coloring problem on computers.

Consider next panels (b3)-(b5) of Fig. 2 plotted similarly for $q=0.25,0.6665$, and 0.999 , but now on the $(\theta, \phi)$-plane. The negative zone is elliptic on panel (b3), whereas it is almost stripe-like on panel (b4) [11]. Although 

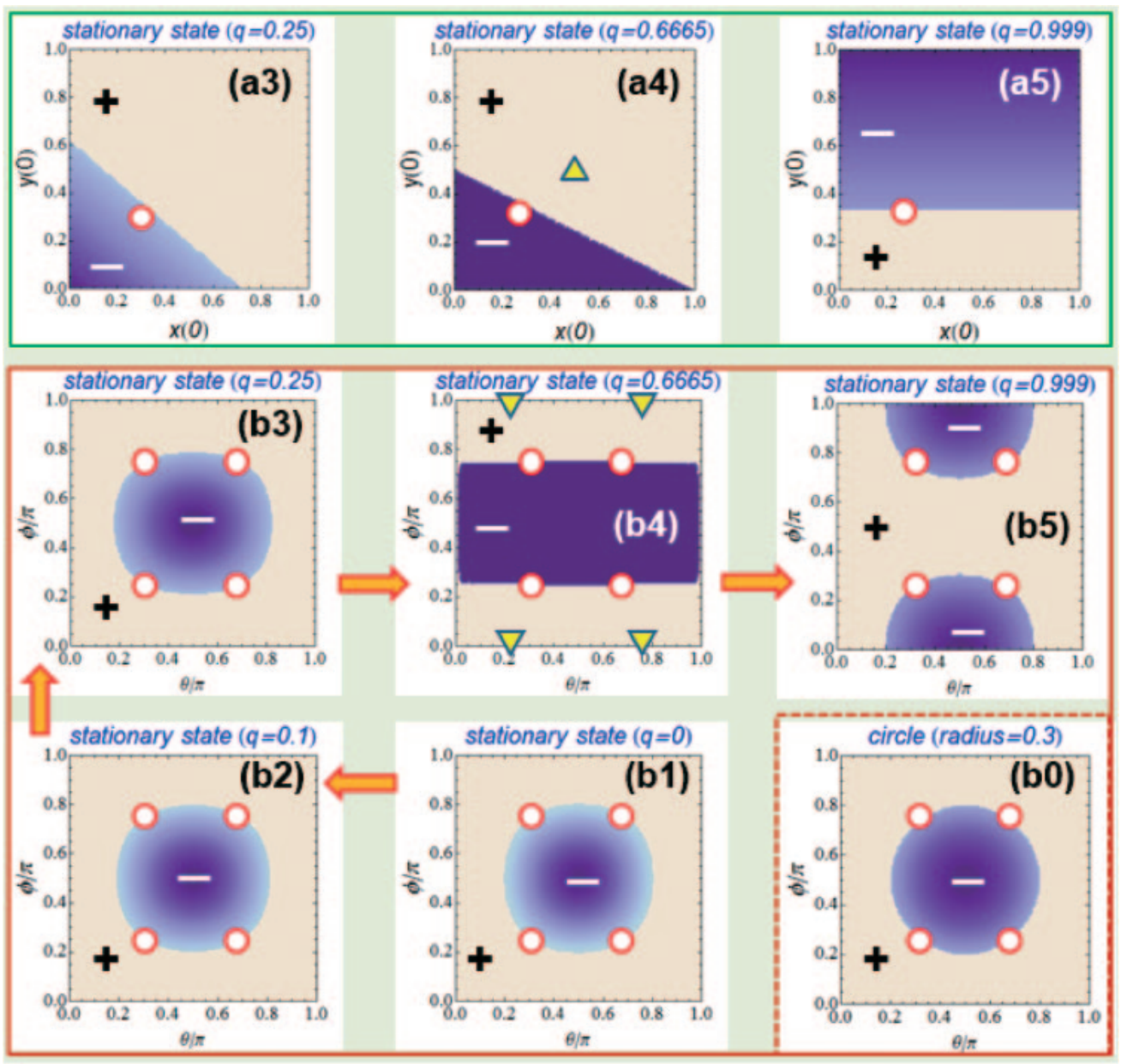

Fig. 2. The region of positive (in brighter color) and negative (in darker bluish color) values of the residual function $f_{1}^{2}(\theta, \phi ; q)$ indicating the stationary states in equilibrium. On the three panels (a3)-(a5) enclosed by the green box, $f_{1}^{2}$ is plotted on the $x(0)-y(0)$ plane for the initial states. On the five panels (b1)-(b5) enclosed by the red box, $f_{1}^{2}$ is plotted on the $(\theta, \phi)$-plane for the initial states. Both $\theta, \phi$ coordinates are normalized by $\pi$. The source probability $q$ is specified on the upper portion of each panel except for panel (b0). The single disk with a red circular boundary on panels (a3)-(a5) and the four disks with respective red circular boundaries on panels (b1)-(b5) indicate the equilibrium stationary states with $x(\infty)=y(\infty)=z(\infty)=1 / 3$.

the negative zone on panel (b5) appears to be split into two parts, it is elliptic as well if it is drawn on the $\phi$-coordinate shifted by a half period. From Eq. (12), let us explicitly find the boundaries for stationary states for the following key values of $q$.

$$
\begin{aligned}
& q=0: \quad \sin ^{2} \theta \sin ^{2} \phi=1 / 3 \\
& q=2 / 3: \quad \sin ^{2} \theta \cos ^{2} \phi=0 \\
& q=1: \quad \sin ^{2} \theta \cos ^{2} \phi=1 / 3 .
\end{aligned}
$$

In Fig. 2, the boundary shown on panel (b1) for $q=0$ and expressed by $\sin ^{2} \theta \sin ^{2} \phi=1 / 3$ in Eq. (13) is almost like a circle. We call this a quasi-circle, which has a certain degree of ellipticity. Likewise, both boundaries shown in panel (b2) for $q=0.1$ and that shown in panel (b3) for $q=0.25$ are quasi-circles. For comparison, consider the following true circle with its center located on the center of the $(\theta, \phi)$-plane and with its radius of $r=0.3$.

$$
\text { (circle): }\left(\frac{\theta}{\pi}-\frac{1}{2}\right)^{2}+\left(\frac{\phi}{\pi}-\frac{1}{2}\right)^{2}=\left(\frac{0.3}{\pi}\right)^{2}
$$

This true circle is displayed on panel (b0) enclosed in a broken red box. Let us examine the series of panels (b1)-(b4) over the range $0 \leq q<2 / 3$ with increasing $q$. We can thus find that the quasi-circle at $q=0$ on panel (b1) accumulates more ellipticity through panels (b2) and (b3) to finally become two lines on panel (b4) for $q=0.6665$. The single-lobe vortex-like structure displayed on panels (b1)-(b3) is in a contrast to two-lobe vortex-like structures in the case of photonic vortices [7].

Exactly at $q=2 / 3$, the initial-state boundary is given by $\sin \theta \cos \phi=0$ from Eq. (13). The solutions to $\sin \theta \cos \phi=$ 0 is $\phi / \pi=1 / 2$, thereby being a single horizontal line in the middle. Its additional solutions $\theta=0$ and $\theta / \pi=1$ are just 
the boundary overlapping the respective vertical boundaries of a panel. That is why we chose to draw on panel (b4) the initial-state boundary for $q=0.6665$, which is slightly smaller than $q=2 / 3$. It is now numerically found that the initial-state boundaries are located approximately at $\phi / \pi=1 / 4$ and $3 / 4$, thus referring respectively to the lower and upper boundaries of the blue stripe on panel (b4).

Upon closer look on panel (b4), the region of $f_{1}^{2}<0$ form a rounded square like a medicine pill, rather than a stripe. Therefore, it is remarkable that a small variation of $q=2 / 3-0.6665 \approx 0.0002$ causes such a drastic change on the initial-state phase space. This is clearly indicative of a first-order phase change. For reference, such a rounded square of the medicine pill is called a lozenge.

For $q=1$ on panel (b5) of Fig. 2, the boundary in Eq. (13) is $\sin ^{2} \theta \cos ^{2} \phi=1 / 3$, which is nothing but the boundary $\sin ^{2} \theta \sin ^{2} \phi=1 / 3$ for $q=0$ but with a phase shift of $\phi / \pi=1 / 2$. Hence, the boundary on panel (b5) of Fig. 2 appears to be broken into two pieces in comparison to its counterpart on panel (b1). From an overall view on panels (b1) through (b5), there is a certain phase change across panel (b4) whereby a circle-like boundaries transit into two halves.

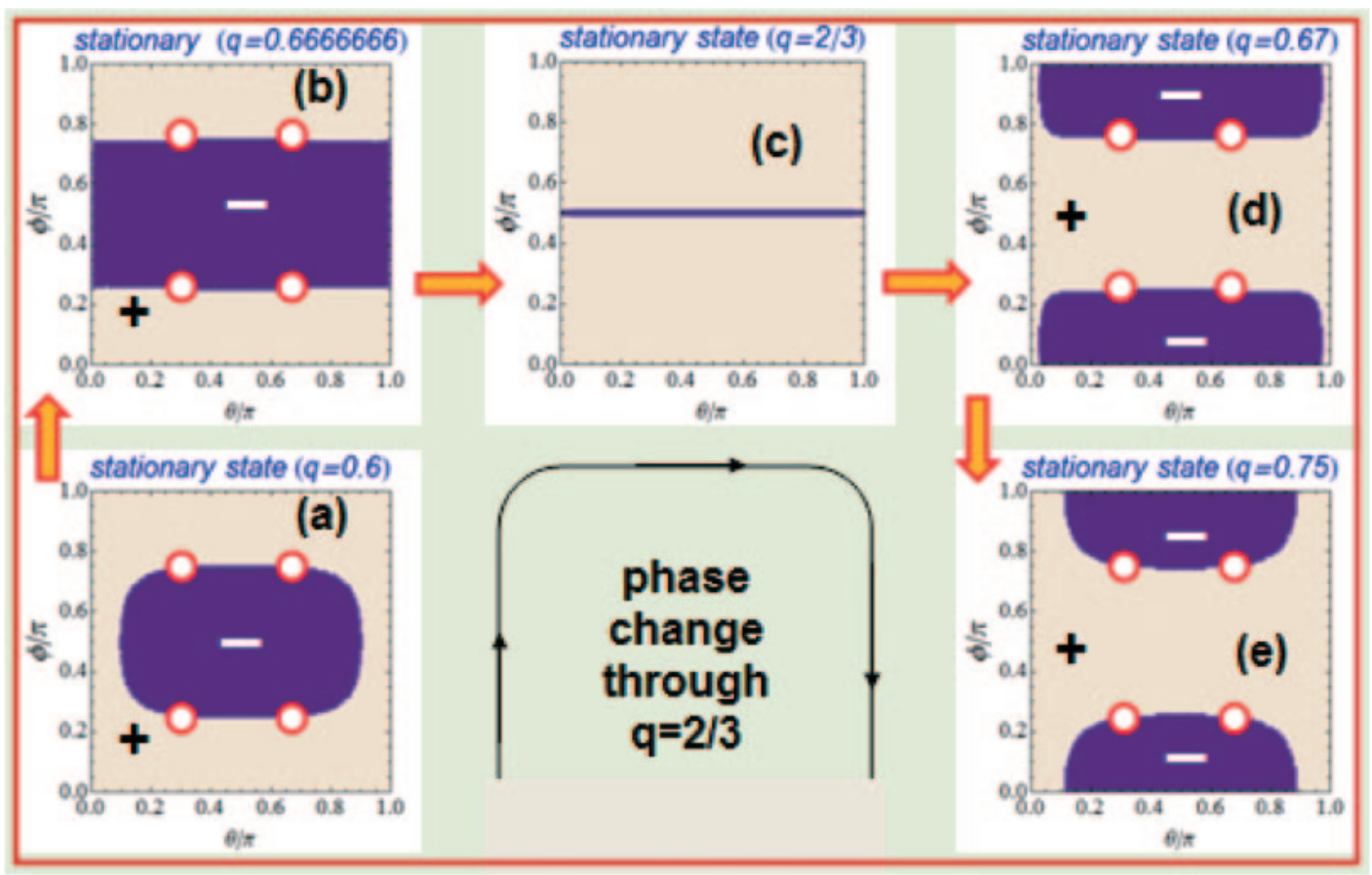

Fig. 3. Plots of $f_{1}^{2}(\theta, \phi ; q)$ near $q=2 / 3$, thus demonstrating an abrupt phase change from one-piece pills through a horizontal line to two halves of a pill. The five panels are arranged in the clockwise direction over (a) $q=0.6$, (b) $q=0.6666666$, (c) $q=2 / 3$, (d) $q=0.67$, and (e) $q=0.75$.

In order to more closely examine the phase change across $q=2 / 3$, we prepared Fig. 3 . As indicated by the interpanel arrows, the five panels of Fig. 3 are arranged in the clockwise direction over (a) $q=0.6$, (b) $q=0.6666666$, (c) $q=2 / 3$, (d) $q=0.67$, and (e) $q=0.75$. First of all, we have exaggerated the horizontal line on panel (c) for $q=2 / 3$. Secondly, for $q=0.6666666$ being closer to $q=2 / 3$ than the previous $q=0.6665$, the blue region of $f_{1}^{2}<0$ on panel (b) of Fig. 3 looks almost like a strip. In contrast, panel (a) for $q=0.6$ clearly exhibits a rounded square of a medicine pill, rather than a stripe. On panel (a), this shape of the initial-state boundary is certainly a quasi-circle with a higher level of ellipticity. In other words, panel (a) is just an extension of panels (b1)-(b3) of Fig. 2, but with an increased ellipticity.

Both panels (d) and (e) of Fig. 3 demonstrate how the linear boundary on panel (c) of Fig. 3 undergoes slow transitions into two halves of a quasi-circle previously displayed on panel (b5) of Fig. 2.

As with Eq. (11), let us express the stationary state $\vec{V}(\infty)$ in terms of two angles $\left(\theta_{\infty}, \phi_{\infty}\right)$ as follows.

$$
\vec{V}(\infty) \equiv\left\{\begin{array}{c}
x(\infty) \\
y(\infty) \\
z(\infty)
\end{array}\right\}=\left\{\begin{array}{c}
\cos ^{2}\left(\theta_{\infty}\right) \\
\sin ^{2}\left(\theta_{\infty}\right) \cos ^{2}\left(\phi_{\infty}\right) \\
\sin ^{2}\left(\theta_{\infty}\right) \sin ^{2}\left(\phi_{\infty}\right)
\end{array}\right\} .
$$

Hence, the equilibrium condition $x(\infty)=y(\infty)=z(\infty)=1 / 3$ is reduced to the two requirements: $\cos ^{2}\left(\theta_{\infty}\right)=1 / 3$ and $\cos ^{2}\left(\phi_{\infty}\right)=\sin ^{2}\left(\phi_{\infty}\right)=1 / 2$. In the domain $\left\{\left(\theta_{\infty}, \phi_{\infty}\right): 0 \leq \theta_{\infty} \leq \pi, 0 \leq \phi_{\infty} \leq \pi\right\}$, we are thus led to the particular set 
of four points with $\theta_{\infty} / \pi \approx 0.304,0.696$ (numerically found) and $\phi_{\infty} / \pi= \pm 1 / 4, \pm 3 / 4$. In Fig. 2 , the corresponding states in terms of $\left(\theta_{\infty}, \phi_{\infty}\right)$ are marked by the four small disks in red circular boundaries on each of panels (b0)-(b5). Therefore, the single small disk in red circular boundary on each of panels (a1)-(a3) on the $x(0)-y(0)$-plane corresponds to these four small disks in the same red circular boundary.

As a further observation on Fig. 2, there is hardly any visible color distribution (namely, almost in the same color) inside the strip-like zone on panel (b4) for $q=0.6665$. On the other hand, we find slight degrees of color distributions in the interiors of the broken quasi-circle on panel (b5) for $q=0.999$. From comparing panels (b1)-(b4) of Fig. 2, we find that the squeezing is horizontally directed [11].

On panels (b1)-(b5) of Fig. 2, $\vec{V}(\infty)$ denoting the final equilibrium lies on the initial-state boundary, which reminds us of the commensurability issue of the vortex lattices or crystalline lattices discussed in conjunction with layered superconductors [4]. Let us take another look at a special off-equilibrium case that $x(0)=y(0)=1 / 2$ with $z(0)=0$, which is marked by the single yellow triangle with the green boundary on panel (a4) of Fig. 2. This special case corresponds to $\theta / \pi=1 / 4$ or $\theta / \pi=3 / 4$ with $\phi / \pi=0$ or $\phi / \pi=1$ [8]. Panel (b4) of Fig. 2 marks these four states by the same yellow triangles with the green boundaries, located on the horizontal boundaries (both on the top and bottom). As expected, these states with the triangular markers do not lie on the initial-state boundary obtained for the equilibrium stationary state.

\section{Phase Change and Criticality}

Consider a little more complicated TPM $T_{2}^{2}$ presented in Eq. (4). Its three eigenvalues are found to be $\left(\lambda_{2}^{2}\right)_{0}=1$ and $\left(\lambda_{2}^{2}\right)_{ \pm}=\frac{1}{2}(1-2 q) \pm \frac{1}{2} \sqrt{\Lambda_{2}^{2}(q)}$ with $\Lambda_{2}^{2}(q) \equiv 1-12 q+32 q^{2}-24 q^{3}$. Instead of analytically finding the bounds on the absolute values of $\left(\lambda_{2}^{2}\right)_{ \pm}$as with Eq. (6) for $T_{1}^{2}$, we plot just numerically $\left|\left(\lambda_{2}^{2}\right)_{ \pm}\right|$in Fig. 4(a) as the source probability $q$ is varied. As a result, it is confirmed that $\left|\left(\lambda_{2}^{2}\right)_{ \pm}\right|<1$ for $0<q<1$. Notice that $\left|\left(\lambda_{2}^{2}\right)_{+}\right|=1$ at both $q=0$ and 1 , whereas $\left|\left(\lambda_{2}^{2}\right)_{-}\right|=1$ only at $q=1$.

Take note in Fig. 4(a) that the two curves are overlapping such that $\left|\left(\lambda_{2}^{2}\right)_{+}\right|=\left|\left(\lambda_{2}^{2}\right)_{-}\right|$over the two regions of $q$ as indicated by the two horizontal broken lines in green color. In fact, these overlapping regions correspond to both $\left(\lambda_{2}^{2}\right)_{ \pm}$ being complex. Complex $\left(\lambda_{2}^{2}\right)_{ \pm}$arise from negative $\Lambda_{2}^{2}(q)$. Outside these overlapping regions, we plot in Fig. 4(b) only the real parts $\left(\lambda_{2}^{2}\right)_{ \pm}$so that they can take negative values around $q=0.6$.

As with Eq. (8), the Markov dynamics $A_{2}^{2}(q)$ for the TPM $T_{2}^{2}$ is broken first into three parts $A_{2}^{2}(q) \equiv$ $\left[A_{2}^{2}(q)\right]_{0}+\left[A_{2}^{2}(q)\right]_{+}+\left[A_{2}^{2}(q)\right]_{-}$. We do not present the detailed procedure for obtaining $A_{2}^{2}(q)$, because it involves so much complicated algebraic manipulations even with the help of commercial symbolic-language softwares such as Mathematica. In particular, both $\left[A_{2}^{2}(q)\right]_{+}$and $\left[A_{2}^{2}(q)\right]_{-}$designating the transient parts are exceedingly complicated. Fortunately, the fact that $\left|\left(\lambda_{2}^{2}\right)_{ \pm}\right|<1$ except at both ends $q=0$ and 1 relieves us of the labor of explicitly finding $\left[A_{2}^{2}(q)\right]_{ \pm}$.

Therefore, we list below the simple stationary part $\left[A_{2}^{2}(q)\right]_{0}$ only.

$$
\left[A_{2}^{2}(q)\right]_{0} \equiv \frac{1}{4-7 q+6 q^{2}}\left(\begin{array}{ccc}
\left(2-5 q+4 q^{2}\right) & \left(2-6 q+8 q^{2}-3 q^{3}\right) & \left(4 q-6 q^{2}+3 q^{3}\right) \\
\left(2-5 q+4 q^{2}\right) & \left(2-6 q+8 q^{2}-3 q^{3}\right) & \left(4 q-6 q^{2}+3 q^{3}\right) \\
\left(2-5 q+4 q^{2}\right) & \left(2-6 q+8 q^{2}-3 q^{3}\right) & \left(4 q-6 q^{2}+3 q^{3}\right)
\end{array}\right) .
$$

As for Eq. (12), we obtain the corresponding residual function $f_{2}^{2}(\theta, \phi ; q)$ from Eq. (16).

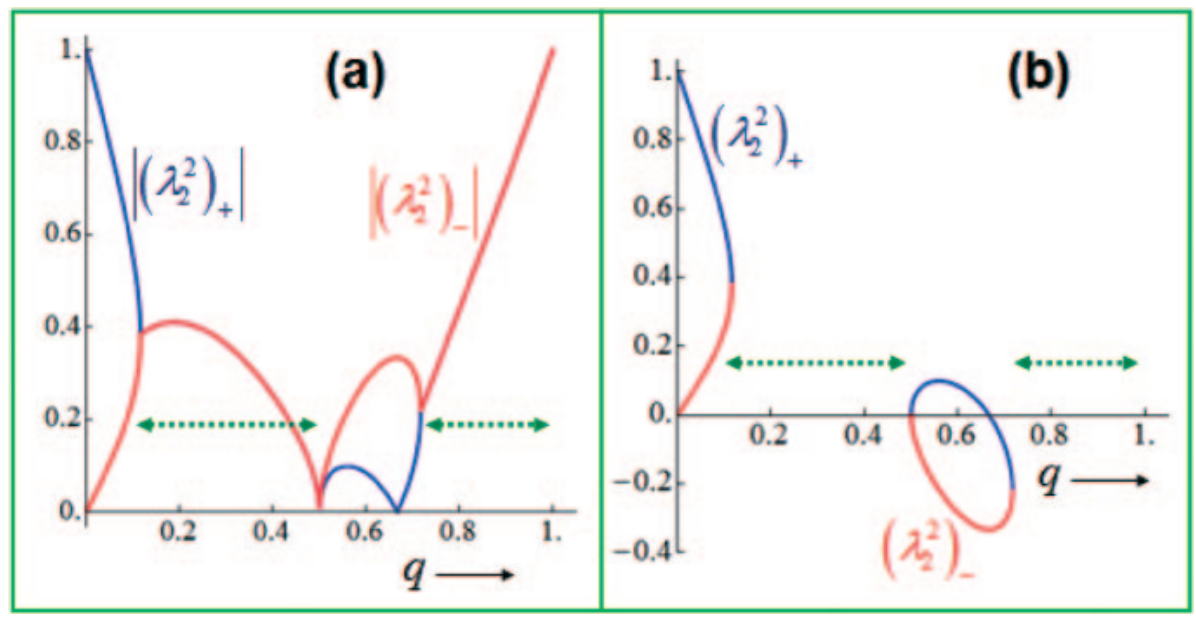

Fig. 4. (a) The absolute values of the eigenvalues $\left|\left(\lambda_{2}^{2}\right)_{+}\right|$in blue color and $\left|\left(\lambda_{2}^{2}\right)_{-}\right|$in red color for the transient parts in case of the TPM $T_{2}^{2}$. Both are drawn as a function of the source probability $q$. (b) Real parts $\left(\lambda_{2}^{2}\right)_{ \pm}$when $\Lambda_{2}^{2}(q)>0$. 


$$
\begin{aligned}
0=f_{2}^{2}(\theta, \phi ; q) \equiv & \left(2-5 q+4 q^{2}\right) \cos ^{2} \theta+\left(2-6 q+8 q^{2}-3 q^{3}\right) \sin ^{2} \theta \cos ^{2} \phi \\
& +\left(4 q-6 q^{2}+3 q^{3}\right) \sin ^{2} \theta \sin ^{2} \phi-\frac{1}{3}\left(4-7 q+6 q^{2}\right) .
\end{aligned}
$$

As with Eq. (13), we list below the initial-state boundaries for the following key values of $q$ [3].

$$
\begin{aligned}
& q=0: \quad \sin ^{2} \theta \sin ^{2} \phi=1 / 3 \\
& q=1 / 3: \quad \cos ^{2} \theta+\sin ^{2} \theta\left(\cos ^{2} \phi+\sin ^{2} \phi\right)=1 \\
& q=2 / 3: \quad\left(1+\sin ^{2} \phi\right) \sin ^{2} \theta=1 \\
& q=1: \quad \cos ^{2} \theta+\sin ^{2} \theta\left(\cos ^{2} \phi+\sin ^{2} \phi\right)=1 .
\end{aligned}
$$

The reason why we write here the trivial identity $\cos ^{2} \theta+\sin ^{2} \theta\left(\cos ^{2} \phi+\sin ^{2} \phi\right)=1$ for $q=1$ is to emphasize that it is satisfied for all combinations of $(\theta, \phi)$. The same thing applies to the case for $q=1 / 3$. This identity is linked to phase change as will be discussed.

For $0<q<1$, we performed a systematic study on $f_{2}^{2}(\theta, \phi ; q)$ by varying $q$ in a continuous way. Figure 5 shows several representative panels for selected values of $q$. The direction of increasing $q$ is indicated by the arrows along the boundary of the figure's box. The way the colors are employed in Fig. 5 is the same as for panels of (b1)-(b5) of Figs. 2 or 3 .

Overall, Fig. 5 displays that those initial-state boundaries undergo rather dramatic changes as $q$ is varied. Firstly, the region with $f_{2}^{2}<0$ is elliptic for $0<q<1 / 3$. As a result, a slight dependence of the boundary shape on $q$ can be seen through Figs. 5(a)-5(b) for the relatively small- $q$ range over $0<q \ll 1 / 3$. If $q$ is taken to imply either doping level or defect concentration, the suitably defined ellipticity varies linearly through Figs. 5(a)-5(b) with such doping level or defect concentration [4].

In contrast, it might seem true throughout $1 / 3<q<2 / 3$ that the region with $f_{2}^{2}>0$ is elliptic as well. In other word, there is an flip-over change in the sign of $f_{2}^{2}$ across $q=1 / 3$. This flip-over can be considered as a sort of phase change. This assertion will be confirmed shortly. Eventually, at $q=2 / 3$ on Fig. 5(e), the region with $f_{2}^{2}>0$ takes a lemon-like shape, which is characterized by two cusps on its top and bottom. Notice however that the transition in the boundary shapes from Figs. 5(c) to 5(d) is considered to be still continuous. This last observation will be disputed shortly.

From $q=2 / 3$ onward up to $q=1$ through Figs. 5(e)-5(g), the region with $f_{2}^{2}>0$ becomes stripe-shaped. However, right at $q=1$ on Fig. 5(h), the initial-state boundary becomes so fuzzy or chaotic that the regions with $f_{2}^{2}>0$ are intermixed with the regions with $f_{2}^{2}<0$ [14]. This fuzzy structure is due to the highly oscillatory dynamics right at $q=1$. This fact is related in turn to the values of $\left|\left(\lambda_{2}^{2}\right)_{ \pm}\right|=1$ at $q=1$, as shown by Fig. 4 .

Such a fuzzy landscape is obtained exactly for $q=1 / 3$ as well (but not presented). This fact is understandable from the fact that $f_{2}^{2}(\theta, \phi ; q)$ reduces to the same expression $\cos ^{2} \theta+\sin ^{2} \theta\left(\cos ^{2} \phi+\sin ^{2} \phi\right)=1$ at both $q=1 / 3$ and $2 / 3$ as listed in Eq. (18). Slightly off $q=1 / 3$, say, for $q=0.3333$ as shown in Fig. 5(c), the initial-state boundary is merely a normal quasi-circle. As a consequence, the above-mentioned flip-over in the sing of $f_{2}^{2}$ is accompanied indeed by a phase change.

Graphically speaking, a two-dimensional quasi-circle is squeezed into a two-dimensional lemon, as it goes from Figs. 5(d) to 5(e). The top and bottom ends of the lemon on Fig. 5(e) open up as we goes towards Fig. 5(f). As a check on the equation for the lemon on Fig. 5(e), consider $\left(1+\sin ^{2} \phi\right) \sin ^{2} \theta=1$ in Eq. (18) for $q=2 / 3$. Unlike the single horizontal line displayed panel (c) of Fig. 3 for the TPM $T_{1}^{2}, q=2 / 3$ for the TPM $T_{2}^{2}$ is not singular as shown by panel (e) of Fig. 5. Therefore, we employed $q=2 / 3$ for Fig. 5 instead of its approximate $q=0.6665$ in Fig. 2.

At the location of the cusps in Fig. $5(\mathrm{e}), \theta / \pi=0.5$. Hence, we obtain two solutions $\phi / \pi=0,1$. As another way of finding representative initial-state boundary points, consider the particular coordinates $\phi / \pi=1 / 4,3 / 4$. From ( $1+$ $\left.\sin ^{2} \phi\right) \sin ^{2} \theta=1$ in Eq. (18), we thus obtain the corresponding coordinates $\theta / \pi=1 / 3,2 / 3$. We marked in Fig. $5(\mathrm{e})$ the four locations $(\theta, \phi)=(1 / 3,1 / 4),(\theta, \phi)=(1 / 3,3 / 4),(\theta, \phi)=(2 / 3,1 / 4)$, and $(\theta, \phi)=(2 / 3,3 / 4)$ by small disks with red circular boundaries. The same set of small disks are placed on the initial-state boundary(ies) for all the panels (a)(g) of Fig. 5.

Finally, we reach a stripe on Fig. 5(g). This stripe represents the extreme anisotropy in contrast to the nearly perfect isotropy of the quasi-circle shown in panel (b1) of Fig. $2[3,4,11]$. On Fig. 5(g) as $q \rightarrow 1$, we found numerically that the initial-state boundaries are located at either $\theta / \pi \approx 0.3$ or $\theta / \pi \approx 0.7$ irrespectively of $\phi$. This stripe is quite different from the indeterminacy of identity $\cos ^{2} \theta+\sin ^{2} \theta\left(\cos ^{2} \phi+\sin ^{2} \phi\right)=1$ found right at $q=1$ as in Eq. (18). To repeat, the indeterminacy manifests itself as a fuzzy landscape in Fig. 5(h). The same indeterminacy occurs at $q=1 / 3$, also as seen from Eq. (18).

In case of the TPM $T_{1}^{2}$, panel (b4) of Fig. 2 or more precisely Fig. 3(c) shows that a strip designating a phase change appears at an intermediate value of $0<q=2 / 3<1$. In contrast, in case of the TPM $T_{2}^{2}$, Fig. 5(h) displays that a phase change shows up at the end value of $q=1$, thereby implying an infinite time in the context of dynamics. Do not forget however that a different phase change (namely, the flip-over) takes place across $q=1 / 3$ in case of the TPM $T_{2}^{2}$.

The transition encompassing the lemon- and stripe-like shapes in Fig. 5 corresponds conceptually to the notion of "squeezing" [15]. For this matter, one angular range for the initial states is narrowed or squeezed in the $\theta$-direction, 

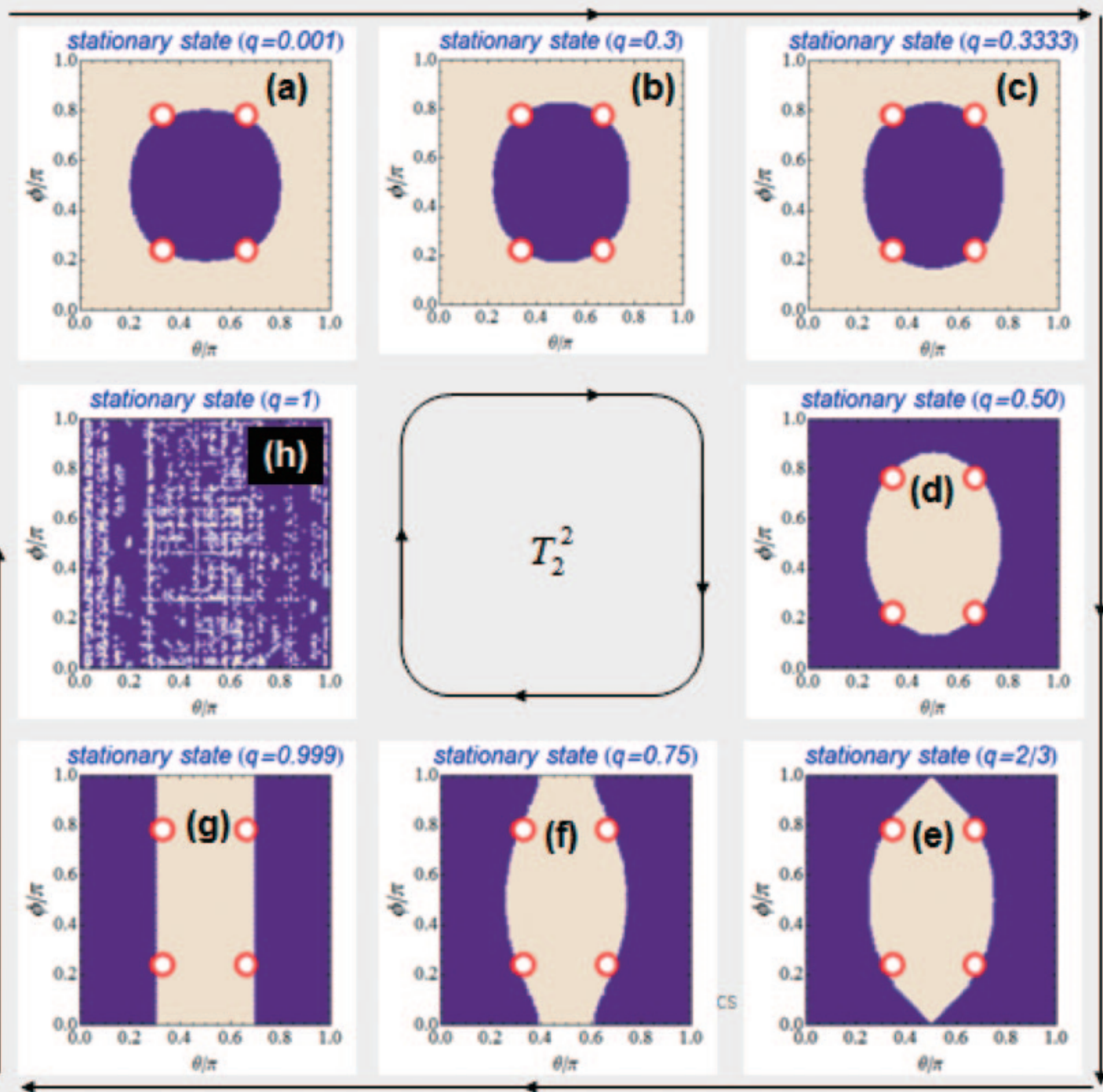

Fig. 5. The initial-state plane is divided into zones with positive (in brighter colors) and negative (in darker bluish colors) values of the residual function $f_{2}^{2}(\theta, \phi ; q)$. The boundaries between two such different zones delineate the initial states, for which the final stationary state is in equilibrium. The initial-state $(\theta, \phi)$-plane is normalized by $\pi$ in both directions. The arrows on the periphery of the box encompassing all the panels indicate the increase in $q$. On each of panels (a) $-(\mathrm{g})$, the set of four small disks in red circular boundaries indicates the equilibrium stationary states as defined in Eq. (15) with $x(\infty)=y(\infty)=z(\infty)=1 / 3$.

whereas the other angular range in the $\phi$-direction slowly gets widened. The stripe in Fig. $5(\mathrm{~g})$ corresponds therefore to an infinite squeezing [11]. So come together the squeezing in one direction and the opening in the other direction. In other words, a directional anisotropy gradually develops as it goes from Figs. 5(a) to 5(g).

\section{Discussions}

We have seen from Eqs. (13) and (18) that the residual functions reduce to the same equation $\sin ^{2} \theta \sin ^{2} \phi=1 / 3$ for $q=0$. Its contour is shown either Figs. 2(b1) or 5(a). These quasi-circles still exhibit certain degrees of ellipticity. In terms of the two variables $(\theta, \phi)$, the true circle defined as in Eq. (14) is quadratically nonlinear, whereas $\sin ^{2} \theta \sin ^{2} \phi=$ $1 / 3$ for the quasi-circles involve a product of two infinite series. As an extension, consider the two-variable twoparameter equation $\sin ^{\alpha} \theta \sin ^{\beta} \phi=1 / 3$ with $\alpha, \beta>0$. We have learned from numerical experiments that the ellipticity increases as either $|\alpha-\beta|$ or $|\alpha+\beta|^{-1}$ increases.

The major axes of the ellipses shown on panels (b1)-(b3) in Fig. 2 are largely oriented in the horizontal direction, whereas the major axes in Fig. 5 are mostly directed in the vertical direction. This geometric asymmetry constitutes the principal feature of quantum squeezing, where the directions of squeezing and anti-squeezing are perpendicular [11]. We have performed a similar analysis for each of the remaining TPMs, namely, for $T_{3}^{2}, T_{4}^{2}$, and $T_{5}^{2}$ mentioned as regards Eq. (4). As a consequence, we found either horizontal or vertical squeezing (but not presented here).

It is noticed from Eq. (11) that vertical stripes with $\theta=0, \pi$ indicate $x(0)=1$ and $y(0)=z(0)=0$, whereas horizontal stripes with $\phi=0, \pi$ imply $x(0)+y(0)=1$ and $z(0)=1$. As a result, stripes refer to relatively pure initial 
states [14], which are relevant to the revival times in the very special adiabatic limit [11]. Here in [11], the initial-state boundaries may refer to the measurement outcomes that are independent of the intensity of the incident illuminating lights after a sequence of complicated optical processes. It is interesting in case of cuprate superconductors that physical stripes are found to undergo changes in spatial orientations as a doping level is varied [16]. Because squeezing refers to the anisotropy, the lemon with a cusp in Fig. 5(e) may imply the start of initial-state squeezing. Other configurations with kinks can also be seen on the parameter plane of position and momentum [14]. Such singularities at the boundaries of the parameter range $0 \leq q \leq 1$ corresponds to the peaking behavior of quantum probability distribution [5] or quantum walks [2].

We have studied the initial-state boundaries so that transitions to the equilibrium stationary state with $x(\infty)=$ $y(\infty)=z(\infty)=1 / 3$ are achieved. The results displayed in Fig. 5 are made for the particular transition probability matrix $T_{2}^{2}$ provided in Eq. (4). In the cases on Figs. 5(a)-5(d) over $0<q<2 / 3$, it is ensured that $0<x(0), y(0), z(0)<$ 1 on each initial-state boundary. Therefore, there could be a certain constraint among three components of the initial state $\vec{V}(0)$. Specially on Fig. 5(e), the two cusps at $\phi=0$ and $\pi$ for $\theta / \pi=1 / 2$ correspond to the initial state with $z(0)=0$ or equally $x(0)+y(0)=1$. Therefore, there could be a constraint imposed only upon the two components (out of three components) of initial state $\vec{V}(0)$.

In the case of two boxes with $g=2$, we could ascribe the origin of the critical value of two thirds $q=2 / 3$ to the ratio of the number of the permutation matrices to the total number of three-by-three components, namely, $q=3 ! / 3^{2}=2 / 3$. Interestingly enough, this magic number $2 / 3$ shows itself up when dealing with the reduction in the visibility or concurrences for two-photon interference experiments $[6,13]$. In addition, the maximum squeezing in two-mode interactions also exhibits a two-third exponent, but in diabatic limits [11].

We remark that the negative-binomial probability has been excluded from this article, since it involves an infinite series in $q$ and hence it makes complicated the interpretation of the ensuing results $[1,16]$. In a future work comparing both positive- and negative-binomial probabilities, we could tell the difference between fermionic and bosonic characteristics when employed for Markov processes [12]. In addition, few-member (more than two) system can be worked out by following the approach taken in this study, although the corresponding algebraic complexity would increase dramatically with certainty $[5,8]$.

If we assume the source probability to be directly proportional to time, we could infer how fast the changes in geometric shapes take place between any two neighboring states as found in Figs. 2, 3, and 5. The last but not the least point is to extend our phase plane $\{(\theta, \phi): 0 \leq \theta \leq \pi, 0 \leq \phi \leq \pi\}$, which is considered to be the irreducible Brillouin zone. By this way, we can discuss an array of vortices and topological physics.

\section{Conclusion}

In summary, we have examined both one- and two-component binomial probabilities by forming appropriate transition matrices for Markovian dynamics. As a result, Markovian dynamics interpreted on the parameter plane designating initial states provides us with quite useful tools for understanding several key physical phenomena. From a geometric viewpoint, the changes observed in the initial-state boundary shapes indicate interesting dynamical evolutions including phase changes.

\section{Acknowledgments}

This research has been supported by Basic Science Research Program through the National Research Foundation (NRF) of Korea funded by the Ministry of Education, Science and Technology (Grant Numbers: NRF-2011-0023612 and NRF-2015R1D1A1A01056698). The authors benefitted greatly from the advice of a reviewer in better shaping this manuscript.

\section{REFERENCES}

[1] Feller, W., An Introduction to Probability Theory, John-Wiley \& Sons, New York (1968).

[2] Kitagawa, T., Rudner, M. S., Berg, E., and Demler, E., "Exploring topological phases with quantum walks," Phys. Rev. A, 82: 033429 (2010).

[3] Moon, K., Mori, H., Yang, K., Girvin, S. M., MacDonald, A. H., Zheng, L., Yoshioka, D., and Zhang, S.-C., "Spontaneous interlayer coherence in double-layer quantum Hall systems: Charged vortices and Kosterlitz-Thouless phase transitions," Phys. Rev. B, 51: 5138-5170 (1995).

[4] Matsuda, M., Fujita, M., Wakimoto, S., Fernandez-Baca, J. A., Tranquada, J. M., and Yamada, K., "Magnetic dispersion of the diagonal incommensurate phase in lightly doped $\mathrm{La}_{2-x} \mathrm{Sr}_{x} \mathrm{CuO}_{4}$, , Phys. Rev. Lett., 101: 197001 (2008).

[5] Kilin, S. Ya., and Krinitskaya, T. B., “Amplitude-phase multistability in multiatomic optical systems," Phys. Rev. A, 48: 3870 (1993).

[6] Franco, R. L., Bellomo, B., Maniscalco, S., and Compagno, G., "Dynamics of quantum correlations in two-qubit systems within non-Markovian environments," Int. J. Mod. Phys. B, 27: 1345053 (2013).

[7] Agarwal, G. S., "Engineering non-Gaussian entangled states with vortices by photon subtraction," New J. Phys., 13: 073008 (2011). 
[8] Delanty, M., Rebić, S., and Twamley, J., "Superradiance and phase multistability in circuit quantum electrodynamics," New J. Phys., 13: 053032 (2011).

[9] Jeltes, McNamara, J. M., Hogervorst, W., Vassen, W., Krachmalnicoff, V., Schellekens, M., Perrin, A., Chang, H., Boiron, D., Aspect, A., and Westbrook, C. I., "Comparison of the Hanbury Brown-Twiss effect for bosons and fermions," Nature, 445: 402-405 (2007).

[10] Dajka, J., Łuczka, J., and Hänggi, P., "Distance between quantum states in the presence of initial qubit-environment correlations: A comparative study," Phys. Rev. A, 84: 032120 (2011).

[11] Gross, C., "Spin squeezing, entanglement and quantum metrology with Bose-Einstein condensates," J. Phys. B: At. Mol. Opt. Phys., 45: 103001 (2012).

[12] Sansoni, L., Sciarrino, F., Vallone, G., Mataloni, P., Crespi, A., Ramponi, R., and Osellame, R., "Two-particle bosonicfermionic quantum walk via integrated photonics," Phys. Rev. Lett., 108: 010502 (2012).

[13] Osorio, C. I., Sangouard, N., and Thew, R. T., "On the purity and indistinguishability of down-converted photons," J. Phys. B: At. Mol. Opt. Phys., 46: 055501 (2013).

[14] Larson, J., "Dynamics of the Jaynes-Cummings and Rabi models: Old wine in new bottles," Phys. Scr., 76: 146 (2007).

[15] Shore, B. W., and Knight, P. L., "The Jaynes-Cummings model,” J. Mod. Optics, 40: 1195-1238 (1993).

[16] Matsuo, K., "Glauber-Sudarshan $P$ representation of negative binomial states," Phys. Rev. A, 41: 519-522 (1990). 